\title{
NITROGEN-DOPED CARBON QUANTUM DOTS WITH PINELLIA TERNATA AS CARBON SOURCE FOR HIGH SENSITIVE DETERMINATION OF CHROMIUM (VI)
}

\author{
DAI, J. ${ }^{1}-$ WANG, Y. $\mathrm{J}^{2 *}$ \\ ${ }^{1}$ College of Biological and Pharmaceutical Engineering, West Anhui University, Luan 237012, \\ Anhui, China \\ ${ }^{2}$ College of Materials and Chemical Engineering, West Anhui University, Luan 237012, Anhui, \\ China \\ *Corresponding author \\ e-mail:3070714927@qq.com \\ (Received 29 ${ }^{\text {th }}$ Apr 2019; accepted $16^{\text {th }}$ Jul 2019)
}

\begin{abstract}
A novel nitrogen-doped fluorescent carbon dots was successfully prepared via a simple onestep hydrothermal method using the medicinal plant Pinellia ternata as an environment-friendly carboncontaining precursor for the first time and ethylenediamine as nitrogen source. The diameter, morphology, ultimate composition and optical performance of the prepared Pinellia ternata nitrogendoped carbon quantum dots (N-CQDs) were characterized by a series of analytical characterization techniques. The photostability was also investigated through the conditions of ionic strength, $\mathrm{pH}$, and storage time. The obtained results showed that the particle size distribution of Pinellia ternata-based NCQDs was uniform with the fluorescence quantum yield as high as $21.3 \%$, and it also exhibited good excitation dependence and excellent light stability. Based on the fluorescence quenching mechanism of fluorescent inner filter effect, it has high sensitivity, selectivity and anti-interference ability for chromium (VI). Under optimal working conditions, the nanoprobe showed a good and sensitive linear response $\left(\mathrm{R}^{2}=0.9955\right)$ toward chromium (VI) within the concentration range of $0 \sim 200 \mu \mathrm{M}$ with a low detection limit of $15 \mathrm{nM}$. This method has been successfully applied to the detection of chromium (VI) in environmental water samples, providing a new idea for the development of green natural compound carbon materials.
\end{abstract}

Keywords: $N$-CQDs, fluorescence quecnching mechanism, fluorescent nanoprobe, $\operatorname{Cr}(\mathrm{VI})$, environmental samples

\section{Introduction}

Chromium is a steel-gray metal. There is no free state chromium in nature. Chromium (II) is a strong reductant, which is extremely unstable and can be rapidly oxidized to chromium (III) (Abdolmohammad-Zadeh and Sadeghi, 2012; Barrera-Díaz et al., 2012). Therefore, it mainly exists in two forms: chromium (III) and chromium (VI). In addition, as one of the most important micronutrient elements in human body, chromium is an important blood sugar regulator and also one of the components of proteolytic enzymes (Bauer et al., 2013; Broadhurst et al., 2006; Sedman et al., 2006). It plays an important role in the metabolism of glucose and lipid and protein synthesis. The toxicity of chromium was related to its valence (Cosata and Klein, 2006; Gagneten and Imhof, 2009). As a component of glucose tolerance factor, activator of some metabolic enzymes and stabilizer of nucleic acid (Ahmad et al., 2011; Costa, 1997; Kim et al., 2015), chromium (III) could promote insulin to play a role, maintain normal glucose metabolism, promote the production of cholesterol and fatty acids and promote hematopoietic function (Vallejos et al., 2012). Chromium (III) deficiency in human 
body could cause diabetes, coronary atherosclerosis and cardiovascular diseases. The toxicity of chromium (VI) was about 100 times higher than that of chromium (III) (Wang et al., 2017). It was also one of the most susceptible metals to allergy. It was second only to nickel, and more easily absorbed and accumulated by human body, causing a series of pathological changes, such as chromium rhinopathy, and even inducing cancer (Ma et al., 2018; Kong et al., 2017; Yuhua et al., 2018). The International Agency for Research on Cancer (IARC) had rated chromium (VI) as a class I carcinogen (Ohira et al., 2015). Therefore, the monitoring of chromium (VI) had attracted the attention of environmental health workers.

Fluorescent carbon quantum dots (CQDs) had been widely used in various research fields due to their advantages of simple preparation, good chemical stability, easy functionalization, good biocompatibility, low toxicity and special optical properties (Wang and Zhou, 2014; Zhang et al., 2014; Zheng et al., 2015; Carrasco et al., 2016). In recent years, many carbon materials had been used to prepare fluorescent carbon quantum dots (Baker and Baker, 2010; Li et al., 2018; Huang et al., 2015). The synthesis of nitrogen-doped carbon quantum dots with excellent optical properties using green natural carbohydrates as precursors had become one of the most popular research topics (Gong et al., 2017; Wang et al., 2018; Gao et al, 2018).

Pinellia ternata is a kind of nourishing medicinal plant. Its raw materials were cheap and easy to obtain. It could be produced on a large scale. Its main bioactive substances included coumarin, alkaloids, amino acids and polysaccharides. Pinellia ternata was rich in carbon, nitrogen and oxygen elements. It was one of the ideal carbon materials for preparing carbon quantum dots (Teixeira da Silva et al., 2017; $\mathrm{Ng}$ et al., 2012; Zheng et al., 2015).

In this paper, Pinellia ternata-based nitrogen-doped fluorescent carbon quantum dots (Pinellia ternata N-CQDs) with blue fluorescence were prepared by a simple hydrothermal method using Pinellia ternata as precursor and ethylenediamine. The fluorescence quantum yield of Pinellia ternata-based N-CQDs was about 21.3\%, which had excellent excitation wavelength dependence and optical stability. Based on the fluorescence quenching mechanism (Gunnlaugsson et al., 2005; Alizadeh et al., 2016; Algar and Krull, 2008; Zhang et al., 2017; Wang et al., 2010), a novel fluorescent probe had been developed for the sensitivity and selectivity detection of chromium (VI). This method could be successfully applied to the rapid detection of environmental water and soil samples, as well as subsequent research.

\section{Experiment}

\section{Materials and apparatus}

Pinellia ternata was purchased from local flower market (Anhui, China); ethylenediamine (EDA) was purchased from Shanghai Aladdin Biochemical Technology Co., Ltd. (Shanghai, China); citric acid, sodium hydrogen phosphate $\left(\mathrm{Na}_{2} \mathrm{HPO}_{4}\right)$ and sodium hydroxide $(\mathrm{NaOH})$ were purchased from Tianjin Zhiyuan Chemical Reagent Co., Ltd. (Tianjin, China). Metal salts mainly include $\mathrm{AlCl}_{3}$, $\mathrm{K}_{2} \mathrm{Cr}_{2} \mathrm{O}_{7}, \mathrm{MgCl}_{2}, \mathrm{ZnCl}_{2}, \mathrm{CuCl}_{2}, \mathrm{CdCl}_{2}, \mathrm{PbCl}_{2}, \mathrm{CrCl}_{3}, \mathrm{CoCl}_{2}, \mathrm{SnCl}_{2}, \mathrm{FeCl}_{3}$ and $\mathrm{FeCl}_{2}$. All solutions were prepared from ultrapure water $\left(18.2 \mathrm{M} \Omega . \mathrm{cm}, 25^{\circ} \mathrm{C}\right)$ of Milli-Q system.

G9800A fluorescence spectrophotometer (Agilent Technologies, USA) was used to determine fluorescence spectrum and its intensity; UV-2600 UV-Visible 
spectrophotometer (Shimadzu, Japan) was used to determine the UV-Visible spectrum; Tecnai G2 F30s-Twin high resolution field emission transmission electron microscopy (FEI, Netherlands) was used to detect particle size and morphology characteristics of nitrogen doped fluorescent quantum dots; TENSOR 27 Fourier transform infrared spectrometer (FT-IR, Bruker, Germany) was used to determine the infrared spectrum and the structure of materials; D8-advance X-ray powder diffractometer (XRD, Bruker, Germany) was used to determine the crystal morphology; Thermo Scientific k-alpha Xray photoelectron spectrometer (Thermo Fisher Scientific Inc. U.S.A.) was used to analyze the proportion of its elements and chemical oxidation state; Z-2000 atomic absorption spectrometer (Hitachi, Japan) was used for the determination of metal ionization; XH-B vortex instrument (Shanghai Hannuo Instrument Co., Ltd., China) was used for vortex blending; 80-2 high-speed centrifuge (Shanghai Surgical Instrument Factory, China) was used for centrifugal filtration; TOP wave microwave digestion device was equipped with PM 60 microwave digestion tank (Jena, Germany) for soil sample digestion; and BPZ-6003 vacuum drying chamber (Shanghai Yiheng Scientific Instrument Co., Ltd., China) was used for vacuum drying.

\section{Preparation of Pinellia ternata-based N-CQDs}

Pinellia ternata-based N-CQDs were prepared by hydrothermal method according to the reported method. The stems of Pinellia ternata were dried naturally at room temperature and ground into fine powder. The powder prepared by weighing $2.0 \mathrm{~g}$ was evenly dispersed in $90 \mathrm{~mL}$ of hot water at $60^{\circ} \mathrm{C}$. Ethanol $(10 \mathrm{~mL})$ and EDA $(1 \mathrm{~mL})$ were added to mixture evenly at constant temperature. The powder was transferred to a PTFE lined autoclave $(200 \mathrm{~mL})$ and heated at $180{ }^{\circ} \mathrm{C}$ for $9 \mathrm{~h}$. After cooling down to room temperature naturally, the bright yellow aqueous solution was centrifuged at $10000 \mathrm{rpm}$ for $15 \mathrm{~min}$ to remove large particles of sediment. The sediment was purified by $0.22 \mu \mathrm{m}$ membrane filtration. The N-CQDs was stored in a refrigerator at $4{ }^{\circ} \mathrm{C}$ and diluted with deionized water to the required concentration. The synthetic reaction diagram of Pinellia ternata N-CQDs is shown in Figure 1.

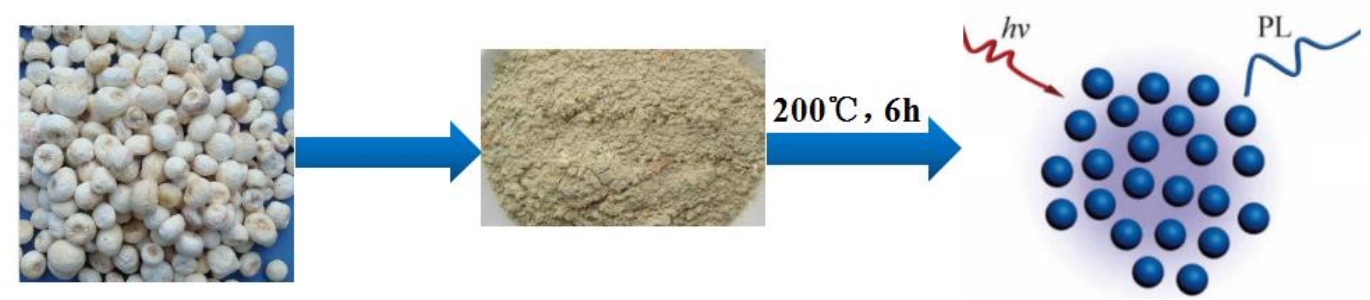

Figure 1. The reaction scheme of the synthesis of the Pinellia ternata-based N-CQDs

\section{Fluorescence quenching test of chromium (VI)}

In $10.0 \mathrm{~mL}$ glass centrifuge tube, $30 \mathrm{~mL}$ water-soluble Pinellia ternata-based $\mathrm{N}$ CQDs solution, $2 \mathrm{~mL}$ pH 7.0 citric acid-disodium hydrogen phosphate buffer solution and different concentration of $\mathrm{Cr}^{6+}$ were added in turn, and then diluted to $4.0 \mathrm{~mL}$ with deionized water. At room temperature, the full eddy mixing reaction lasts for $10 \mathrm{~min}$. The slit width of excitation and emission on the fluorophotometer is $5 \mathrm{~nm}$, the excitation wavelength is lambda ex =356 $\mathrm{nm}$, and the emission wavelength is lambda $\mathrm{em}=444 \mathrm{~nm}$. In order to evaluate the influence of coexisting ions on the fluorescence 
intensity of N-CQDs and to evaluate the selectivity of $\mathrm{Cr}^{6+}$, other metal ions (including $\mathrm{Zn}^{2+}, \mathrm{Cu}^{2+}, \mathrm{Al}^{3+}, \mathrm{Mg}^{2+}, \mathrm{Cd}^{2+}, \mathrm{Pb}^{2+}, \mathrm{Cr}^{3+}, \mathrm{Sn}^{2+}, \mathrm{Co}^{2+}, \mathrm{Hg}^{2+}, \mathrm{Fe}^{3+}$ and $\mathrm{Fe}^{2+}$ ) were detected under the same procedure.

\section{Pretreatment of environmental water and soil samples}

In this study, three different water samples (tap water, lake water and waste water) were selected for analysis and detection. All water samples were filtered by simple centrifugal filtration to remove solid particles and suspended solids, then filtered by $0.22 \mu \mathrm{m}$ membrane, and stored in a $4{ }^{\circ} \mathrm{C}$ refrigerator for storage. In this study, three different soil samples (parking lot, highway) were selected for analysis and detection. Soil samples of $1.0 \mathrm{~g}$ were weighed and added with $1 \mathrm{~mL}$ nitric acid and $3 \mathrm{~mL}$ hydrochloric acid. They were placed in a microwave digestion tank of polytetrafluoroethylene (PTFE). Microwave digestion was carried out at $140{ }^{\circ} \mathrm{C}$ for $15 \mathrm{~min}$, then cooled to room temperature. The digestion was diluted to $50 \mathrm{~mL}$ and stored in a refrigerator at $4{ }^{\circ} \mathrm{C}$.

\section{Results and discussion}

\section{Characterization of Pinellia ternata-based N-CQDs}

In order to further study the nanostructure characteristics of Pinellia ternata-based $\mathrm{N}-\mathrm{CQD}$, the morphology and particle size of Pinellia ternata-based N-CQDs were characterized by transmission electron microscopy (TEM). As shown in Figure 2, the Pinellia ternata-based N-CQDs were uniformly dispersed without agglomeration and were spherical carbon nanoparticles. In addition, the particle size distribution of biomass-based N-CQDs is narrow, mainly between 5 and $6 \mathrm{~nm}$, and the average particle size is about $5.2 \mathrm{~nm}$.

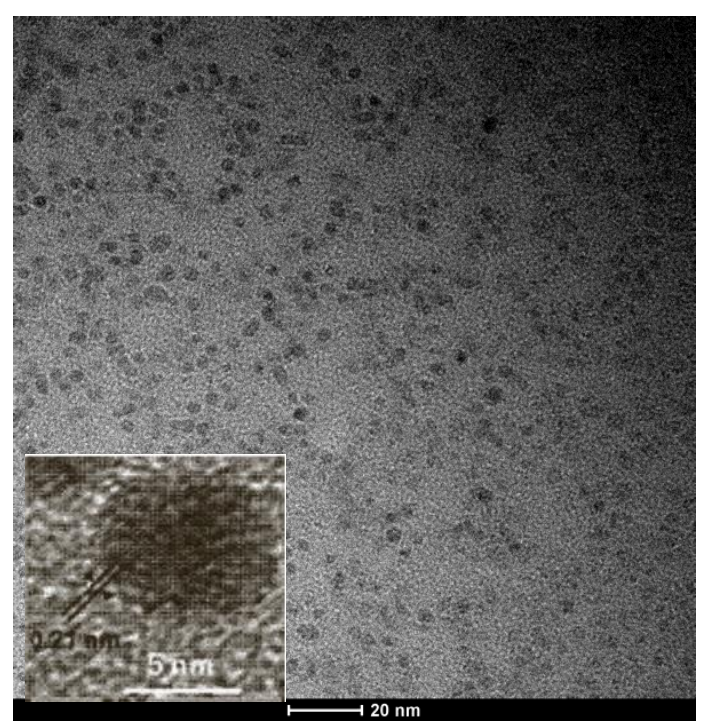

Figure 2. The TEM image of the synthesized N-CQDs

The composition and surface chemical structure of Pinellia ternata-based N-CQDs were determined by Fourier transform infrared spectroscopy (FT-IR) and X-ray electron 
spectroscopy (XPS). Figure 3 is the infrared spectra of N-CQDs. The broad and strong absorption peaks at $3442 \mathrm{~cm}^{-1}$ are attributed to the stretching vibration absorption peaks of $\mathrm{O}-\mathrm{H}$ and $\mathrm{N}-\mathrm{H}$; the peak at $1637 \mathrm{~cm}^{-1}$ is attributed to the stretching vibration absorption peak of $\mathrm{O}=\mathrm{C}-\mathrm{NH}$; the absorption peaks at $1467 \mathrm{~cm}^{-1}$ are attributed to the stretching vibration peak of $\mathrm{COOH}$; and the absorption peaks at $1119 \mathrm{~cm}^{-1}$ and $1078 \mathrm{~cm}^{-1}$ are attributed to the $\mathrm{C}-\mathrm{N}$ bend. Bending vibration peak and $\mathrm{C}-\mathrm{O}$ stretching vibration peak. The results show that the Pinellia ternata N-CQDs have abundant hydrophilic functional groups, such as hydroxyl, carboxyl and carbonyl groups, which have good water solubility and broad application prospects.

The elements of Pinellia ternata-based N-CQDs were characterized by XPS, as shown in Figure 4. XPS elemental analysis is consistent with FT-IR results, which proves that the functional groups containing oxygen and nitrogen, such as hydroxyl, carboxyl, amino groups, etc.

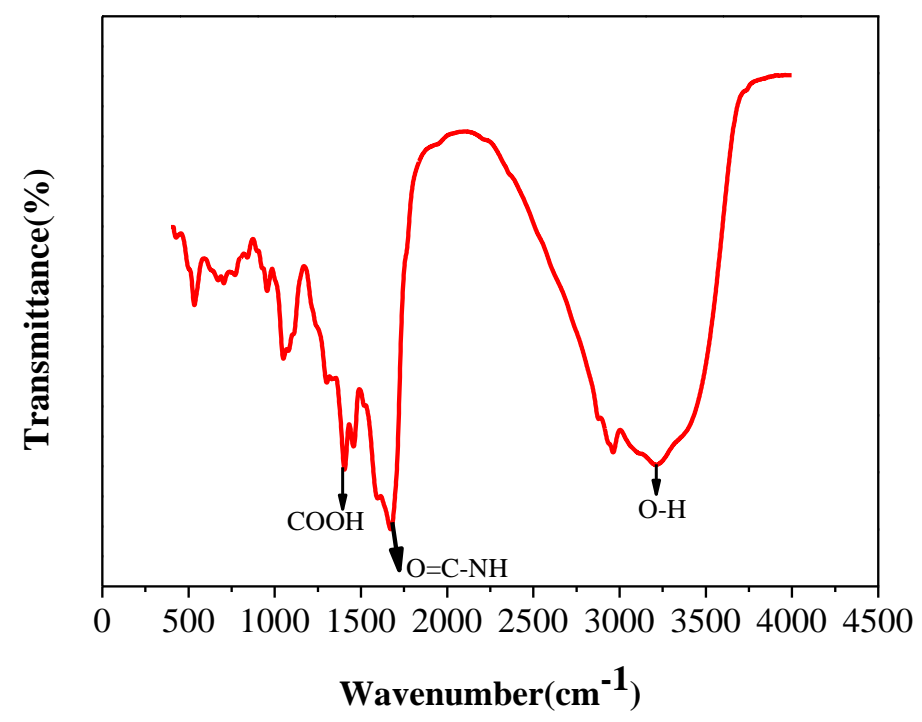

Figure 3. The FT-IR spectrum of the Pinellia ternata-based N-CQDs

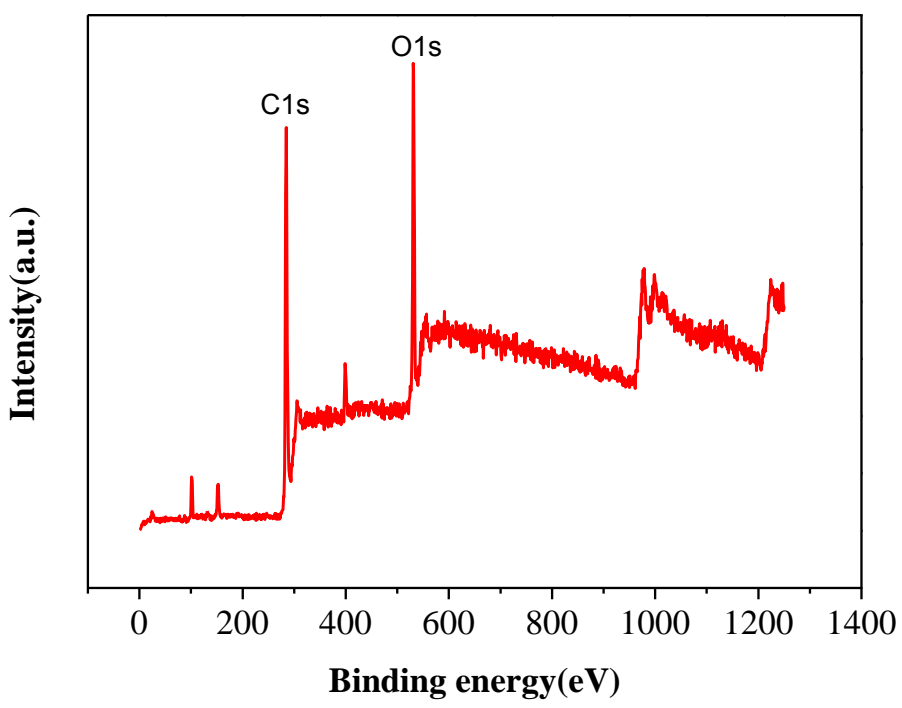

Figure 4. The XPS spectra of the synthesized N-CQDs 


\section{Optical properties of Pinellia ternata-based N-CQDs}

The optical properties of Pinellia ternata-based N-CQDs were characterized by UVVis absorption spectra and fluorescence emission spectra. In addition, the illustrations in Figure 5A show that Pinellia ternata-based N-CQDs are golden in natural light (left) and bright blue fluorescence in $365 \mathrm{~nm}$ ultraviolet light (right). Benefiting from its narrow size distribution, Pinellia ternata-based N-CQDs exhibit relatively symmetrical fluorescence spectra, as shown in Figure $5 B$. Under $356 \mathrm{~nm}$ excitation light, the maximum emission wavelength is $444 \mathrm{~nm}$. The principle of fluorescence depends mainly on the bandgap transition formed by conjugated pion electrons and the surface defects of fluorescent carbon quantum dots. Figure $5 C$ is the fluorescence emission spectra of Pinellia ternata-based N-CQDs at different excitation wavelengths, showing good excitation wavelength-dependent fluorescence behavior. The results shown that nitrogen doping can adjust the intrinsic electronic and surface properties of CQDs, enhance their effective surface defects and internal carbon core structure, and thus significantly improve their optical properties.
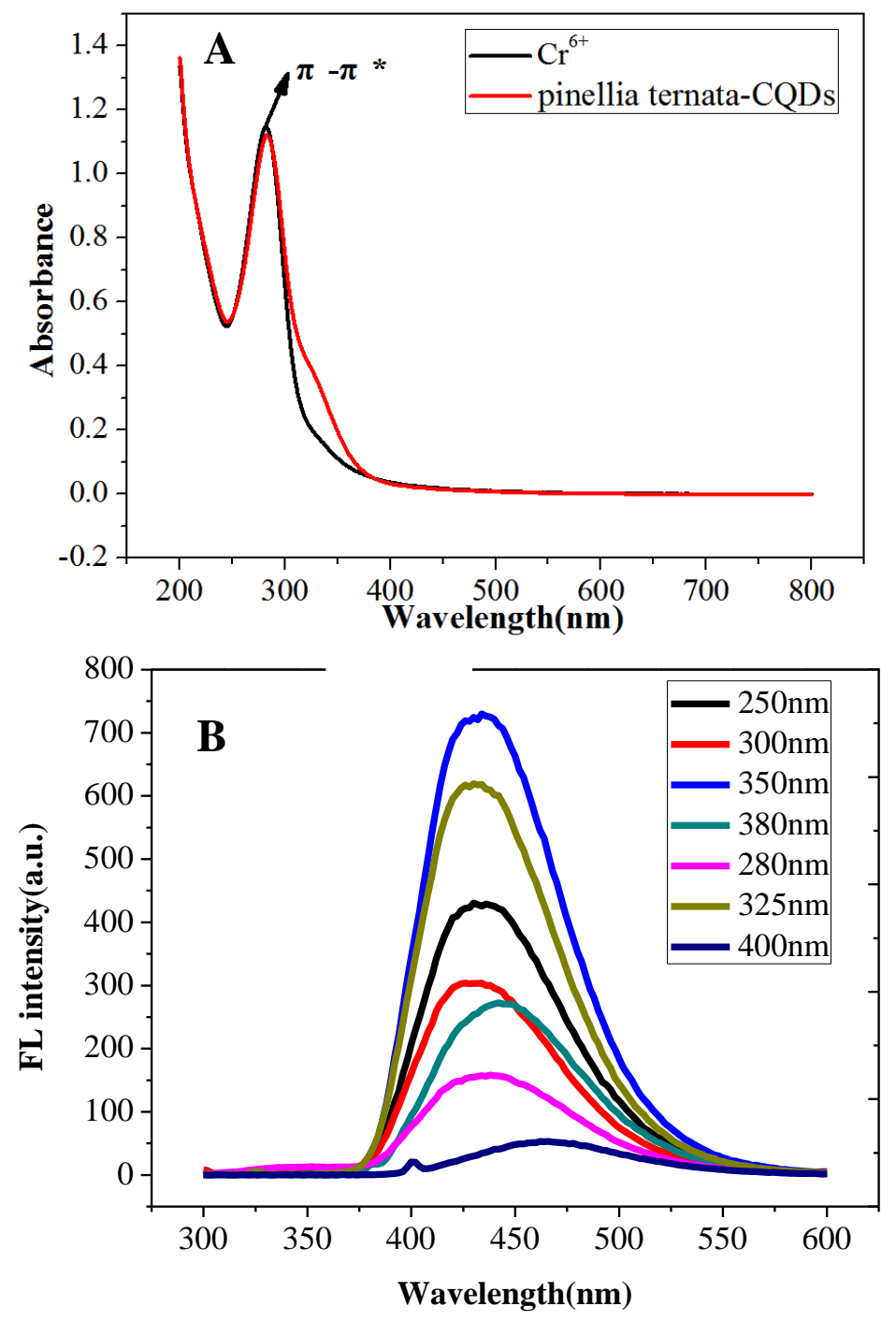

Figure 5. UV absorption spectra of the Pinellia ternata-based $\mathrm{N}-\mathrm{CQDS}$ and $\mathrm{Cr}^{6+}(\mathrm{A})$; fluorescence excitation and emission spectra of the Pinellia ternata-based $N$-CQDs $(B)$ 


\section{Photostability of Pinellia ternata-based N-CQDs}

In order to explore the anti-interference ability of Pinellia ternata-based N-CQDs to the external environment, the light stability test was carried out, and the conditions of ion strength, $\mathrm{pH}$ and storage time were investigated. As shown in Figure 6A, when the $\mathrm{pH}$ value of citric acid-disodium hydrogen phosphate buffer changes from 2.2 to 8.0, its fluorescence intensity does not change obviously under acidic and neutral conditions of $\mathrm{pH}$ 2.2-7.0, but decreases sharply under alkaline conditions of $\mathrm{pH} 8.0$, which indicates that the probe can be used as a fluorescent nanoprobe in acidic medium. Figure $6 B$ investigated the effect of ion strength on the fluorescence intensity of Pinellia ternatabased N-CQDs. As can be seen from the figure, the fluorescence intensity only fluctuates slightly with the increasing concentration of $\mathrm{NaCl}$ solution, and there is no obvious change. Therefore, Pinellia ternata-based N-CQDs still have strong stability in high ionic strength environment. As shown in Figure $6 C$ and D, Pinellia ternata-based $\mathrm{N}-\mathrm{CQD}$ with the same concentration were stored in refrigerator at room temperature for $3 \mathrm{~h}$ or even $4{ }^{\circ} \mathrm{C}$ for 3 months under the same experimental conditions. The fluorescence intensity of Pinellia ternata-based N-CQDs remained basically unchanged and stable, and no floating or sediment could be seen in its aqueous solution by naked eyes. The above experimental results show that the Pinellia ternata-based N-CQDs have excellent optical stability.
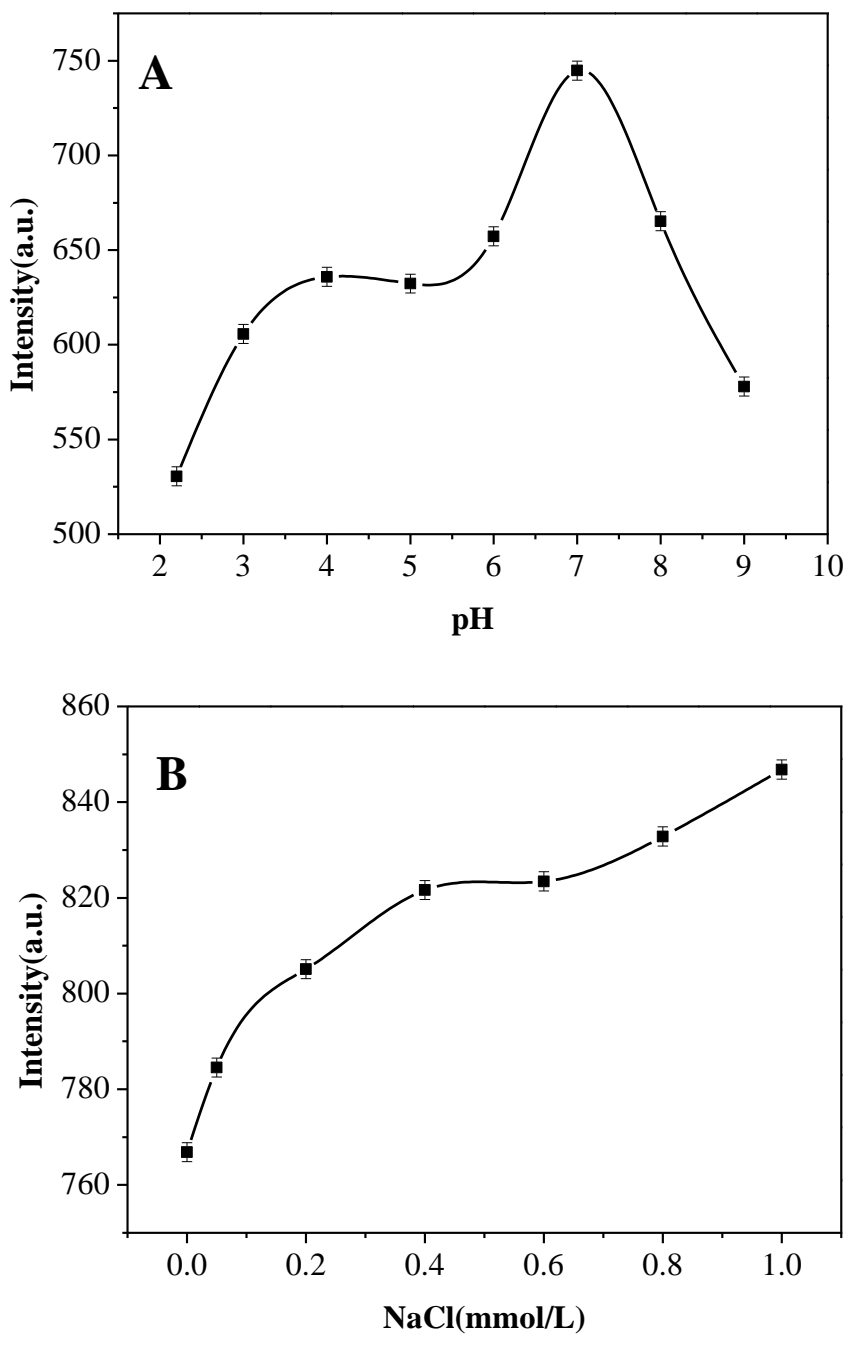


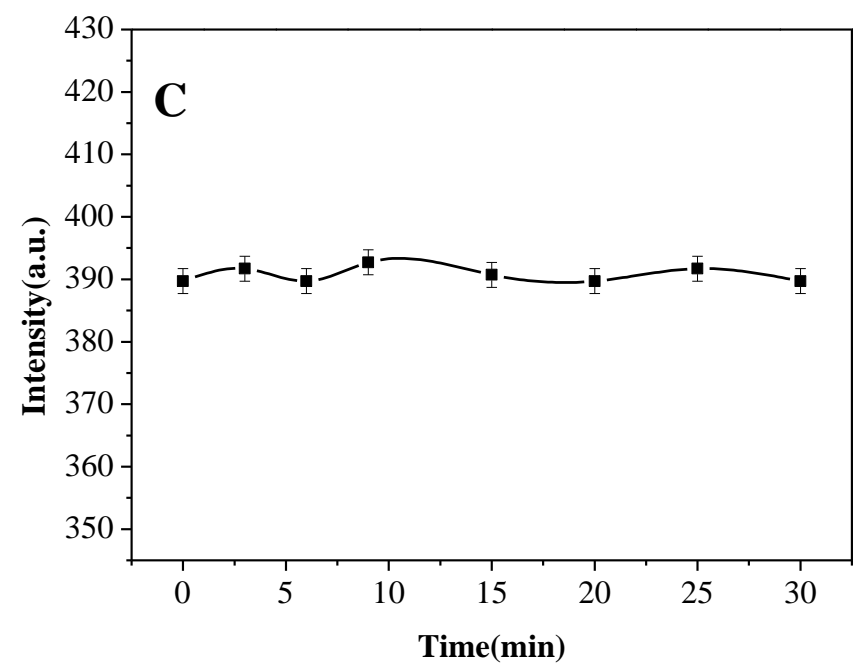

Figure 6. Effects of various conditions on the fluorescence intensity of the Pinellia ternatabased $\mathrm{N}$-CQDs: (A) $\mathrm{pH}$; (B) NaCl concentrations; (C) storage time

\section{Selective investigation test}

In order to investigate the selectivity of Pinellia ternata-based N-CQD fluorescence intensity to different metal ions, 12 metal ions with the same concentration of $\mathrm{Al}^{3+}$, $\mathrm{Mg}^{2+}, \mathrm{Zn}^{2+}, \mathrm{Cu}^{2+}, \mathrm{Cr}^{6+}, \mathrm{Cr}^{3+}, \mathrm{Cd}^{2+}, \mathrm{Co}^{2+}, \mathrm{Sn}^{2+}, \mathrm{Ag}^{+}, \mathrm{Pb}^{2+}, \mathrm{Fe}^{3+}, \mathrm{Fe}^{2+}$, were investigated. As shown in Figure 7, only $\mathrm{Cr}^{6+}$ can effectively quench the fluorescence intensity of Pinellia ternata-based N-CQDs, while the introduction of other metal ions does not cause significant changes in the fluorescence intensity of Pinellia ternata-based NCQDs. This indicates that Pinellia ternata-based N-CQDs have high selectivity to $\mathrm{Cr}$ (VI) and can be used as nanoprobe for fluorescence quenching detection of $\mathrm{Cr}$ (VI). In addition to $\mathrm{Cr}^{6+}$, other metal ions will coexist with the actual samples. Therefore, this study further investigated the ion interference problem in the detection of $\mathrm{Cr}^{6+}$ through competitive experiments, that is, in the presence of other metal ions, the detection of $\mathrm{Cr}^{6+}$ was carried out. As shown in Figure 7, the experimental results show that the presence of interfering ions has little effect on the detection of $\mathrm{Cr}^{6+}$. In particular, the potential interference ion $\mathrm{Cr}^{3+}$, although causing a slight reduction in the fluorescence intensity of the detection of $\mathrm{Cr}^{6+}$, has a negligible effect, and can be used to distinguish between $\mathrm{Cr}^{3+}$ and $\mathrm{Cr}^{6+}$, which is a characteristic that some fluorescent nanoprobes for the detection of $\mathrm{Cr}^{6+}$ do not possess. In conclusion, Pinellia ternata-based N-CQDs have good selectivity and anti-interference ability as a fluorescent nanoprobe for detection of $\mathrm{Cr}(\mathrm{VI})$.

\section{Optimization of experimental conditions}

The fluorescence quenching mechanism can be used for rapid detection of $\mathrm{Cr}$ (VI). In order to obtain the best fluorescence response signal, some key experimental factors such as solution $\mathrm{pH}$ and reaction time were further investigated and optimized. Firstly, the fluorescence intensity of Pinellia ternata-based N-CQDs was studied in different $\mathrm{pH}$ ranges (2.0-12.0). It can be seen from Figure $8 A$ that the fluorescence quenching efficiency is the highest when the $\mathrm{pH}$ value is 7.0. The influence of $\mathrm{pH}$ value on the quenching process may be related to the form and distribution of acid-base equilibrium 
groups on Pinellia ternata N-CQDs surface and Cr (VI) in solution (Vaz et al., 2017). $\mathrm{Cr}(\mathrm{VI})$ exhibits a $\mathrm{pH}$-dependent dynamic equilibrium in aqueous solution: $2 \mathrm{CrO}_{4}{ }^{2-}$ $+2 \mathrm{H}^{+} \rightleftharpoons \mathrm{Cr}_{2} \mathrm{O}_{7}{ }^{2-}+2 \mathrm{H}_{2} \mathrm{O}$. $\mathrm{Cr}(\mathrm{VI})$ mainly exists in the form of chromium $\left(\mathrm{CrO}_{4}^{2-}\right)$ and dichromate $\left(\mathrm{Cr}_{2} \mathrm{O}_{7}^{2-}\right)$. Although it has the same charge number, it has higher molar volume, so it has lower electron density and lower effective interaction with Pinellia ternata-based N-CQDs. Its fluorescence quenching efficiency is lower than that of chromite $\left(\mathrm{CrO}_{4}{ }^{2-}\right)$. In acidic medium, $\mathrm{Cr}$ (VI) mainly exists in the form of dichromate $\left(\mathrm{Cr}_{2} \mathrm{O}_{7}{ }^{2-}\right)$, so its fluorescence quenching efficiency is low, and gradually increases with the increase of $\mathrm{pH}$.

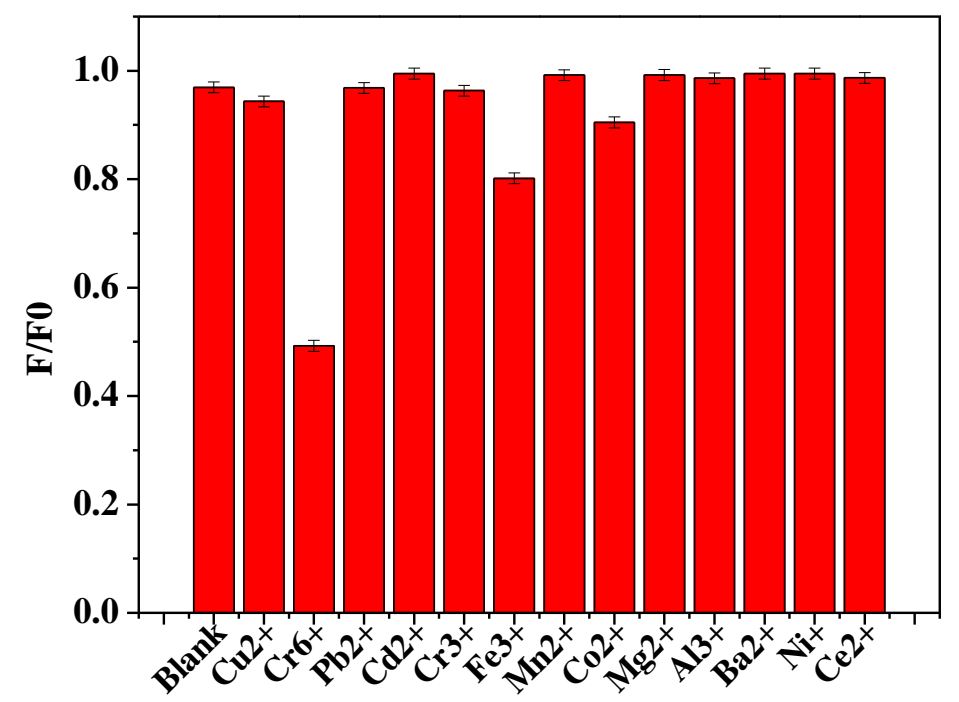

Figure 7. Evaluation of method selectivity against possible interferents

In addition, in alkaline medium, the oxygen-containing functional groups on Pinellia ternata-based N-CQDs are mainly in the form of chromate $\left(\mathrm{CrO}_{4}{ }^{2-}\right)$, which is easy to dephosphorize and increase the negative charge on the surface. The interaction between $\mathrm{Cr}$ (VI) and negatively charged chromite $\left(\mathrm{CrO}_{4}{ }^{2-}\right)$ is weakened due to electrostatic repulsion, which shows that the fluorescence quenching efficiency begins to decrease gradually. Therefore, $\mathrm{pH} 7.0$ is defined as the optimum determination condition.

In order to determine the quenching response time of $\mathrm{Cr}^{6+}$ to Pinellia ternata $\mathrm{N}$ CQDs, the reaction time was also discussed in this study. Figure $8 B$ shows the fluorescence intensity of Pinellia ternata N-CQDs with time after adding $\mathrm{Cr}^{6+}$. The fluorescence intensity of Pinellia ternata-based N-CQDs decreases sharply in 0-2 min, and reaches the lowest fluorescence intensity in $2 \mathrm{~min}$. Later, the fluorescence intensity of Pinellia ternata-based N-CQDs decreases slightly but tends to stabilize gradually with the extension of time. Therefore, the optimal fluorescence quenching response time is $2 \mathrm{~min}$.

\section{Study on methodological performance}

Under the optimum experimental conditions, the relationship between the fluorescence quenching intensity of Pinellia ternata N-CQDs and the concentration of $\mathrm{Cr}^{6+}$ was studied, and a quantitative analysis method for the fluorescence quenching of $\mathrm{Cr}^{6+}$ was established. As shown in Figure 9A, the fluorescence intensity of N-CQDs 
decreases gradually with the increase of the concentration of $\mathrm{Cr}^{6+}$ and changes regularly, which further proves the feasibility and application prospects of N-CQDs as a fluorescent probe for the detection of $\mathrm{Cr}^{6+}$. As shown in Figure 9B, the fluorescence quenching efficiency $(\mathrm{F} 0 / \mathrm{F})$ has a good linear relationship with the concentration of $\mathrm{Cr}^{6+}$ in the range of $0-200 \mu \mathrm{M}$. The linear equation is $\mathrm{F} 0 / \mathrm{F}=1.0218+0.1156 \mathrm{C}\left(\mathrm{Cr}^{6+}\right)$, $\mathrm{R}^{2}=0.9955$, and the detection limit is $15 \mathrm{nM}(\mathrm{S} / \mathrm{N}=3)$.
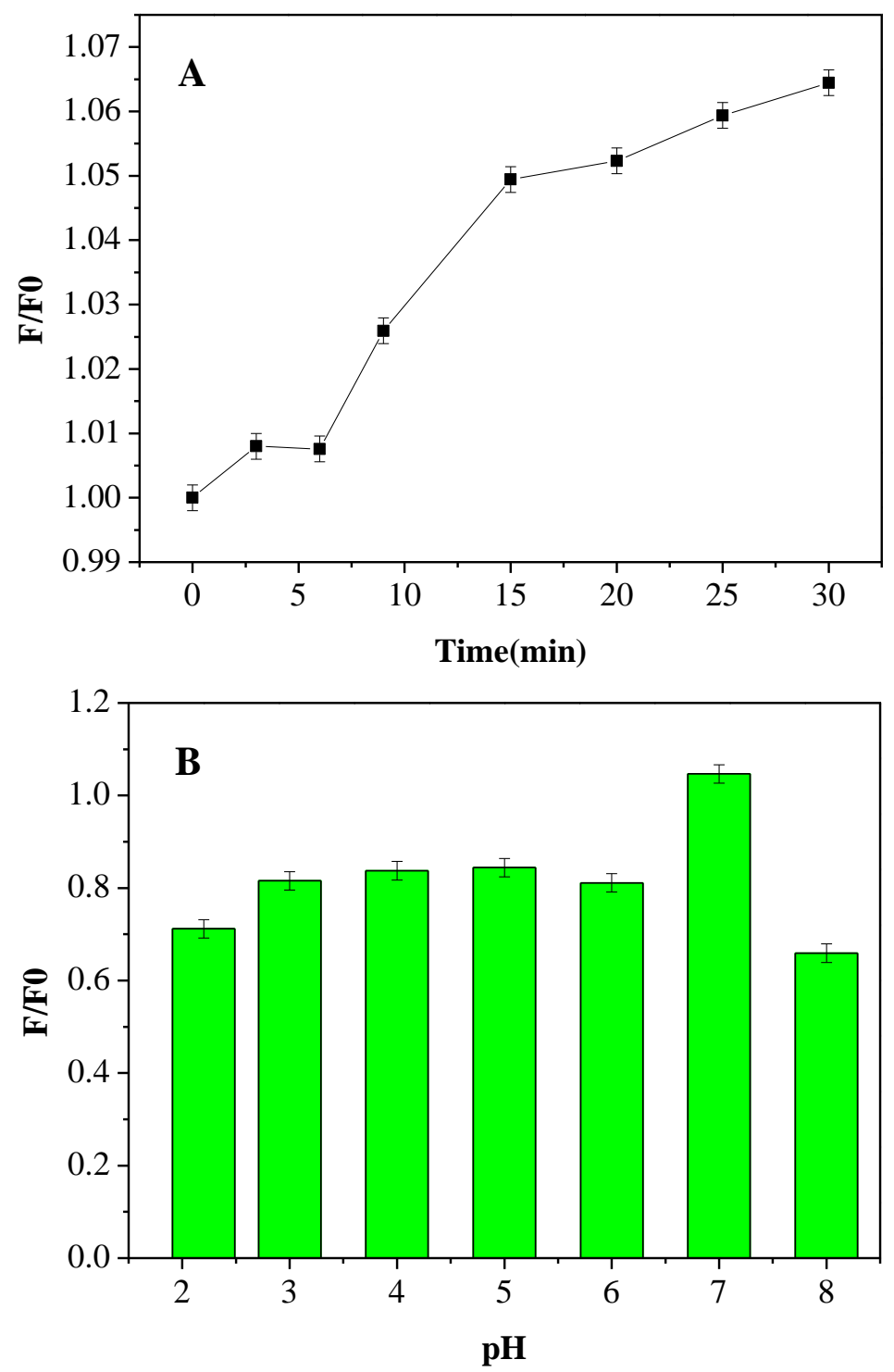

Figure 8. Effect of reaction time $(A)$ and $p H(B)$ on the fluorescence intensity of the Pinellia ternata-based $N$-CQDs

\section{Analysis of fluorescence quenching mechanism}

The fluorescence quenching mechanism of Pinellia ternata-based N-CQDs for detecting chromium (VI) is similar to that of polyacrylamide-based N-CQDs for detecting $\mathrm{Hg}^{2+}$. It is caused by the synergistic effect of fluorescent inner filter effect (IFE) and electron transfer. As shown in Figure 10, at $\mathrm{pH} 7.0$, the ultraviolet-visible absorption spectra of $\mathrm{Cr}$ (VI) show two absorption peaks at 272 and $371 \mathrm{~nm}$, 
respectively, while Pinellia ternata-based N-CQDs have ultraviolet absorption peaks at $337 \mathrm{~nm}$, and the maximum excitation and emission wavelengths are 356 and $444 \mathrm{~nm}$, respectively. It is obvious that the absorption spectra of $\mathrm{Cr}$ (VI) overlap with the excitation and absorption spectra of Pinellia ternata-based N-CQDs. Therefore, Cr (VI) partially shields the excitation spectra of Pinellia ternata-based N-CQDs and partially absorbs the light emitted by Pinellia ternata-based N-CQDs, resulting in the fluorescence endofiltration effect between Pinellia ternata-based N-CQDs and $\mathrm{Cr}^{6+}$, resulting in the quenching of the fluorescence of Pinellia ternata-based N-CQDs. In addition, $\mathrm{Cr}^{6+}$ has strong electron acceptance and affinity, and it is easy to capture electrons. When the excited state molecule of Pinellia ternata-based N-CQDs collides with $\mathrm{Cr}^{6+}$, the nitrogen and oxygen functional groups on its surface can combine with $\mathrm{Cr}^{6+}$ to form complexes, which lead to electron transfer and non-radiative transition, leading to fluorescence quenching.
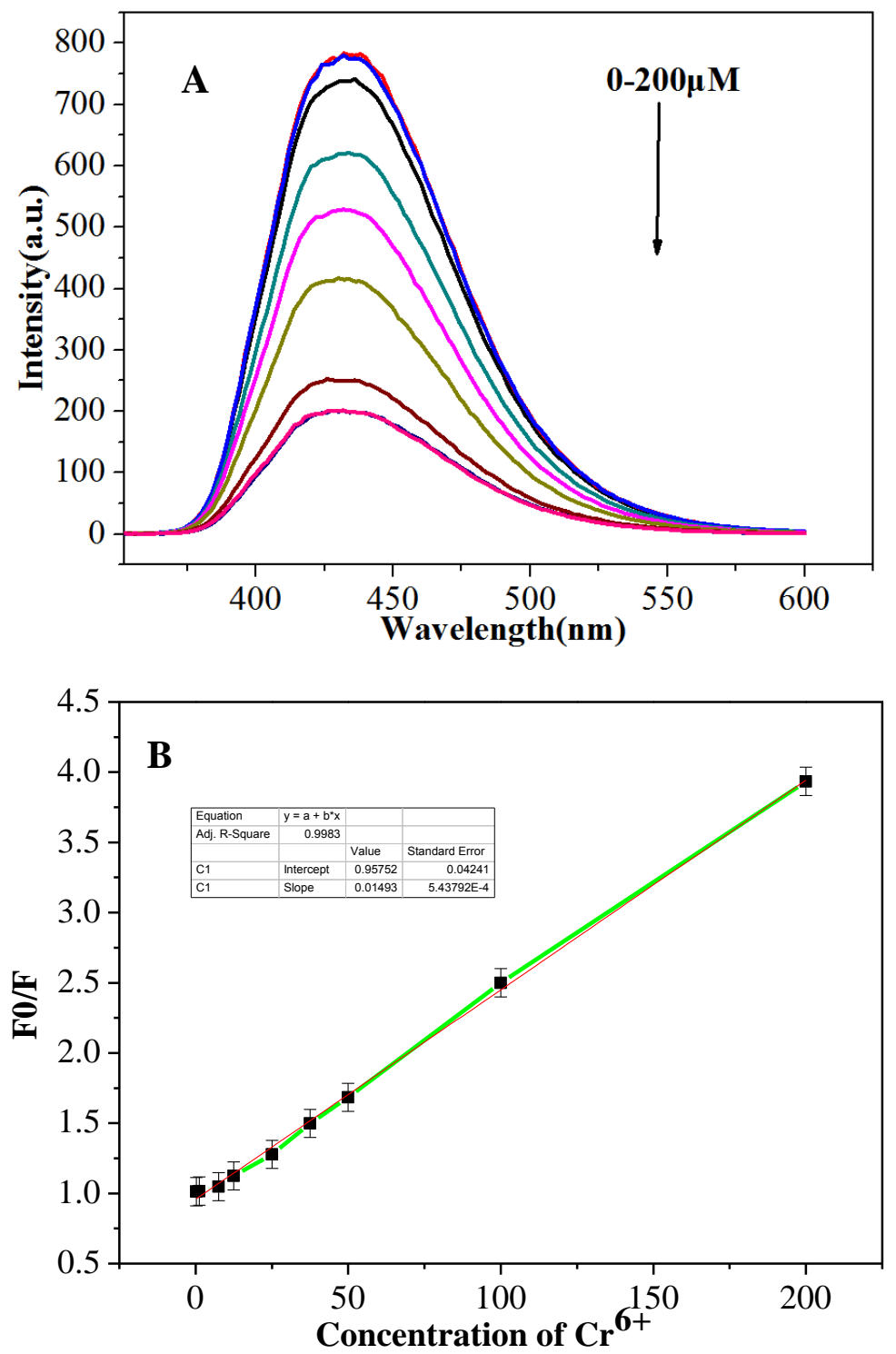

Figure 9. The fluorescence response of the Pinellia ternata-based $\mathrm{N}$-CQDs upon the addition of different concentrations of $\mathrm{Cr}^{6+}(A)$ and a linear correlation of $F 0 / F$ values versus the concentration of $\mathrm{Cr}^{6+}$ over the range from 0 to $200 \mu \mathrm{M}(\mathrm{B})$ 


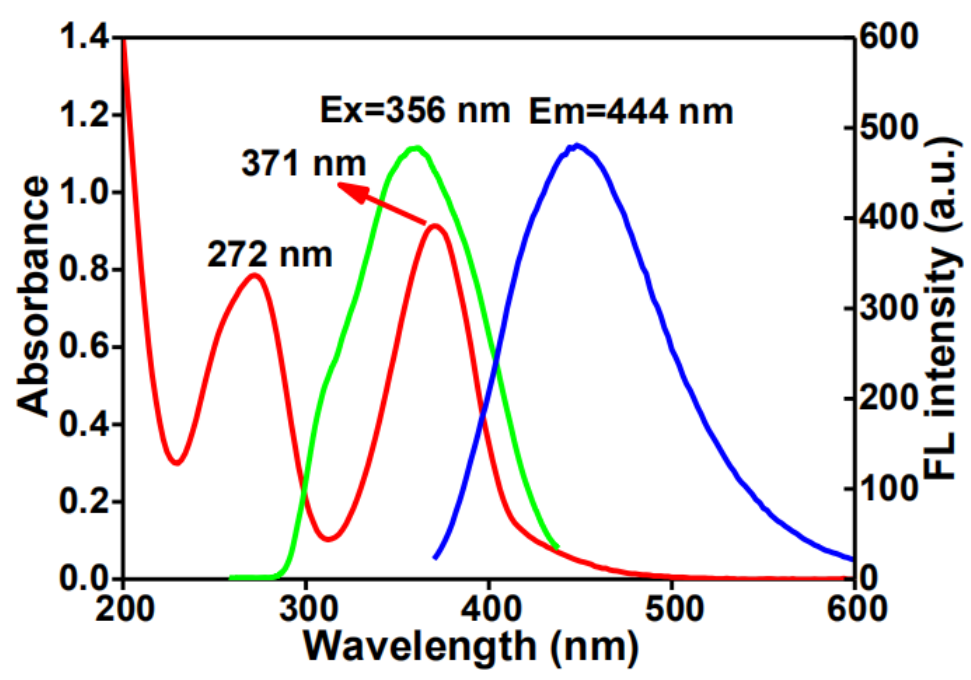

Figure 10. UV-Vis absorption spectra of Cr (VI) and fluorescence excitation/emission spectra of the Pinellia ternata-based $N-C Q D s$

\section{Detection of actual samples}

In order to verify the practicability of this method, the content of $\mathrm{Cr}^{6+}$ in different water samples was investigated. As shown in Table 1, the recoveries of water samples were $94.2-102.5 \%$ and RSD was less than $5.8 \%$. In order to evaluate the accuracy of this method, FAAS method was used to detect the content of $\mathrm{Cr}$ in water samples with the same concentration. The results show that in Table 1, the results of the two methods are very close, so, this method can be used for the rapid analysis and detection of $\mathrm{Cr}^{6+}$ in environmental water samples, and can be effectively applied to further research.

Table 1. Analytical results of $\mathrm{Cr}(\mathrm{VI})$ in the water samples $(n=3)$

\begin{tabular}{c|c|c|c|c|c}
\hline Samples & $\begin{array}{c}\text { Spiked } \\
(\boldsymbol{\mu M})\end{array}$ & $\begin{array}{c}\text { Found } \\
(\boldsymbol{\mu M})\end{array}$ & $\begin{array}{c}\text { Recovery } \\
(\boldsymbol{\%})\end{array}$ & $\mathbf{R S D}(\boldsymbol{\%}, \mathbf{n}=\mathbf{3})$ & $\begin{array}{c}\text { FAAS } \\
\text { Found }(\boldsymbol{\mu M})\end{array}$ \\
\hline \multirow{3}{*}{ Tap water } & 0 & - & - & - & - \\
& 5 & 4.71 & 94.2 & 4.3 & 4.82 \\
& 20 & 19.3 & 96.5 & 3.6 & 19.3 \\
\hline \multirow{3}{*}{ Lake water } & 0 & - & - & - & - \\
& 5 & 4.92 & 98.4 & 5.8 & 4.98 \\
& 20 & 20.5 & 102.5 & 2.5 & 20.1 \\
\hline \multirow{3}{*}{ Waste water } & 0 & - & - & - & - \\
& 5 & 5.10 & 102.0 & 5.3 & 5.04 \\
& 20 & 20.05 & 100.3 & 4.7 & 19.75 \\
\hline
\end{tabular}

“_" not detected

\section{Conclusion}

In this study, for the first time, Pinellia ternata was used as a carbon precursor and ethylenediamine as a nitrogen source. Nitrogen-doped fluorescent carbon quantum dots with excellent optical properties and fluorescent quantum yield of $21.3 \%$ were prepared 
by a simple one-step hydrothermal method. The morphology, particle size, crystalline form, chemical element composition and optical properties were characterized by TEM, FT-IR, XPS, UV-Vis and FS. The results show that Pinellia ternata N-CQDs have uniform size distribution, rich oxygen and nitrogen functional groups on their surface, and show good excitation wavelength dependence and tunability. Based on the quenching mechanism of fluorescence internal filter effect, $\mathrm{Cr}^{6+}$ has high sensitivity, selectivity and anti-interference ability. It can be used as a fluorescent nanoprobe for the analysis and detection of $\mathrm{Cr}^{6+}$ in complex environmental samples. The method has good linear relationship, fast reaction, simple operation, high sensitivity, high selectivity and low detection limit. It provides a new idea for developing carbon quantum dot fluorescent nanoprobe for detecting $\mathrm{Cr}^{6+}$ and has broad application prospects.

Acknowledgements. The subject is supported by the Fund of Nature Science Research Project of Anhui Province (1808085QB33).

\section{REFERENCES}

[1] Abdolmohammad-Zadeh, H., Sadeghi, G. H. (2012): A nano-structured material for reliable speciation of chromium and manganese in drinking waters, surface waters and industrial wastewater effluents. - Talanta 94: 201-208.

[2] Ahmad, M. K., Syma, S., Mahmood, R. (2011): Cr (VI) induces lipid peroxidation, protein oxidation and alters the activities of antioxidant enzymes in human erythrocytes. - Biological trace Element Research 144: 426-435.

[3] Algar, W. R., Krull, U. J. (2008): Quantum dots as donors in fluorescence resonance energy transfer for the bioanalysis of nucleic acids, proteins, and other biological molecules. - Analytical \& Bioanalytical Chemistry 391(5): 1609-1618.

[4] Alizadeh, A., Ghouzivand, S., Khodaei, M. M., et al. (2016): An interesting spectroscopic method for chromofluorogenic detection of cyanide ion in aqueous solution: Disruption of intramolecular charge transfer (ICT). - Journal of Chemical Sciences 128(4): 537-543.

[5] Baker, S. N., Baker, G. A. (2010): Luminescent carbon nanodots: emergent nanolights. Angew. Chem. Int. Ed. 49: 6726-6744.

[6] Barrera-Díaz, C. E., Lugo-Lugo, V., Bilyeu, B. (2012): A review of chemical, electrochemical and biological methods for aqueous $\mathrm{Cr}(\mathrm{VI})$ reduction. - J. Hazard. Mater. 1(12): 223-224.

[7] Bauer, G., Neouze, M.-A., Limbeck, A. (2013): Dispersed particle extraction. A new procedure for trace element enrichment from natural aqueous samples with subsequent ICP-OES analysis. - Talanta 103: 145-152.

[8] Broadhurst, C. L., Domenico, P. (2006): Clinical studies on chromium picolinate supplementation in diabetes mellitus. A review. - Diabetes Technol Ther 8: 677-87.

[9] Carrasco, P. M., Garcia, I., Yate, L., Zaera, R. T., Cabanero, G., Grande, H. J., Ruiz, V. (2016): Graphene quantum dot membranes as fluorescent sensing platforms for $\mathrm{Cr}(\mathrm{VI})$ detection. - Carbon 109: 658-665.

[10] Costa, M. (1997): Toxicity and carcinogenicity of $\mathrm{Cr}(\mathrm{VI})$ in animal models and humans. - Critical Reviews in Toxicology 27: 431-442.

[11] Costa, M., Klein, C. B. (2006): Toxicity and carcinogenicity of chromium compounds in humans. - Critical Reviews in Toxicology 36: 155-163.

[12] Gagneten, A. M., Imhof, A. (2009): Chromium (Cr) accumulation in the freshwater crab, Zilchiopsis collastinensis. - Journal of Environmental Biology 30: 345-348.

[13] Gao, Y., Jiao, Y., Lu, W., Liu, Y., Han, H., Gong, X., Xian, M., Shuang, S. and Dong, C. (2018): Carbon dots with red emission as a fluorescent and colorimeteric dual-readout 
probe for the detection of chromium(VI) and cysteine and its logic gate operation. Mater. Chem. B 38: 1-10.

[14] Gong, X. J., Liu, Y., Yang, Z. H., Shuang, S. M., Zhang, Z. Y., Dong, C. (2017): An “onof-on" fluorescent nanoprobe for recognition of chromium(VI) and ascorbic acid based on phosphorus/nitrogen dual-doped carbon quantum dot. - Anal. Chim. Acta 968: 5-96.

[15] Gunnlaugsson, T., Ali, H. D. P., Glynn, M., et al. (2005): Fluorescent photoinduced electron transfer (PET) sensors for anions. From design to potential application. - Journal of Fluorescence 15(3): 287-299.

[16] Huang, S., Qiu, H. N., Zhu, F. W., Lu, S. Y., Xiao, Q. (2015): Graphene quantum dots as on-of-on fluorescent probes for chromium(VI) and ascorbic acid. - Microchim. Acta 182: 1723-1731.

[17] Kim, H., Lee, B.-I., Byeon, S.-H. (2015): The inner filter effect of $\mathrm{Cr}(\mathrm{VI})$ on Tb-doped layered rare earth hydroxychlorides: new fluorescent adsorbents for the simple detection of $\mathrm{Cr}(\mathrm{VI})$. - Chem. Commun. 51: 725-728.

[18] Kong, W., Wu, D., Li, G., Chen, X., Gong, P., Sun, Z., Chen, G., Xia, L., You, J., Wu, Y. (2017): A facile carbon dots based fluorescent probe for ultrasensitive detection of ascorbic acid in biological fluids via non-oxidation reduction strategy. - Talanta 165: 677-684.

[19] Li, Y. K., Yang, T., Chen, M. L., Wang, J. H. (2018): Supported carbon dots serve as high-performance adsorbent for the retention of trace cadmium. - Talanta 180: 18-24.

[20] Ma, X. Y., Chena, Y., Liua, J., Hana, Y., Ma, S., Chen, X. (2018): Ratiometric fluorescent detection of chromium(VI) in real samples based on dual emissive carbon dots. - Talanta. 185(1): 249-257.

[21] Ng, T. B., Liu, J., Wong, J. H., Ye, X., Wing, S. C., Tong, Y., Zhang, K. Y. (2012): Review of research on dendrobium candidum, a prized folk medicine. - Appl. Microbiol. Biotechnol. 93(5) 1795-1803.

[22] Ohira, S. I., Nakamura, K., Shelor, C. P., Dasgupta, P. K., Toda, K. (2015): Simultaneous electrodialytic preconcentration and speciation of chromium(III) and chromium(VI). Anal. Chem. 87: 11575-11580.

[23] Sedman, R. M., Beaumont, J. A. Y., McDonald, T. A., Reynolds, S., Krowech, G., Howd, R. (2006): Review of the evidence regarding the carcinogenicity of hexavalent chromium in drinking water. - J. Environ. Sci. Heal. C 24: 155-182.

[24] Teixeira da Silva, J. A., Ng, T. B. (2017): The medicinal and pharmaceutical importance of Dendrobium candidum species. - Appl. Microbiol. Biotechnol. 101(6) 2227-2239.

[25] Vallejos, S., Munoz, A., Garcia, F. C., Serna, F., Ibeas, S., Garcia, J. M. (2012): Methacrylate copolymers with pendant piperazinedione-sensing motifs as fluorescent chemosensory materials for the detection of $\mathrm{Cr}(\mathrm{VI})$ in aqueous media. - J. Hazard. Mater. 227-228: 480-483.

[26] Vaz, R., Bettini, J., Júnior, J. G. F., et al. (2017): High luminescent carbon dots as an ecofriendly fluorescence sensor for $\mathrm{Cr}(\mathrm{VI})$ determination in water and soil samples. - Journal of Photochemistry \& Photobiology A Chemistry 346: 502-511.

[27] Wang, H. S., Xie, Y., Bi, J., Li, Y., Song, Y., Cheng, S., Li, D., Tan, M. (2018): Facile one-step synthesis of highly luminescent $\mathrm{N}$-doped carbon dots as efficient fluorescence probe for chromium (VI) detection based on inner filter effect. - New, J. Chem. 5: 1-8.

[28] Wang, L., Zhou, H. S. (2014): Green synthesis of luminescent nitrogen-doped carbon dots from milk and its imaging application. - Anal. Chem. 86: 8902-8905.

[29] Wang, M., Zhang, G., Zhang, D., et al. (2010): Fluorescent bio/chemosensors based on silole and tetraphenylethene luminogens with aggregation-induced emission feature. Journal of Materials Chemistry 20(10): 1858-1867.

[30] Wang, W., Bai, H., Li, H., Lv, Q., Wang, Z., Zhang, Q. (2017): Disposable plastic electrode for electrochemical determination of total chromium and hexavalent chromium. - J. Electroanal. Chem. 794: 148-155. 
[31] Yuhua, Z., Xian, F., Hong, Z., Li, Z. (2018): A highly sensitive and selective detection of $\mathrm{Cr}(\mathrm{VI})$ and ascorbic acid based on nitrogen-doped carbon dots. - Talanta 181: 318-325.

[32] Zhang, H. J., Chen, Y. L., Liang, M. J., Xu, L. F., Qi, S. D., Chen, H. L., Chen, X. G. (2014): Solid phase synthesis of highly fluorescent nitrogen-doped carbon dots for sensitive and selective probing ferric ions in living cells. - Anal. Chem. 86: 9846-9852.

[33] Zhang, H. Y., Wang, Y., Xiao, S., et al. (2017): Rapid detection of Cr(VI) ions based on cobalt(II)-doped carbon dots. - Biosensors \& Bioelectronics 87: 46-52.

[34] Zheng, Q., Qiu, D., Liu, X., Zhang, L., Cai, S., Zhang, X. (2015): Antiproliferative effect of Dendrobium candidum catenatum Lindley polypeptides against human liver, gastric and breast cancer cell lines. - Food Funct. 6(5) 1489-1495.

[35] Zheng, X. T., Ananthanarayanan, A., Luo, K. Q., Chen, P. (2015): Glowing graphene quantum dots and carbon dots: properties, syntheses, and biological applications. - Small 11: $1620-1636$. 